\title{
Semiconductor Materials for Ultrafast Photoswitches
}

\author{
J.-L. Coutaz \\ Laboratory of Microwaves and Characterization, LAHC - University of Savoie
}

73376 Le Bourget du Lac Cedex, France

\begin{abstract}
This paper gives a review of semiconductor materials that are used to fabricate ultrafast photoswitches. The optoelectrical response of the switches is first described with simple models, from which the material requirements are deduced. The basic principles of the required material properties - ultrashort free carrier lifetime and high mobility, high dark resistivity, and high field breakdown - are explained. Then, the most popular ultrafast semiconductors are listed, together with their characteristics. A special emphasis is put on low-temperature grown GaAs. Finally, two applications of these ultrafast materials are presented, namely antennae for terahertz radiation and all-optical nonlinear devices.
\end{abstract}

PACS numbers: 85.60.-q, 85.60.Dw, 72.20.-i, 72.40.+w, 72.80.Ey, 42.65.Re

\section{Introduction}

The laser exhibits two remarkable features, among others, namely its light beam coherence and the possibility of producing very short light pulses. The coherence makes the laser light obeying to the Poisson statistics, leading to weak noise in the transmission of information through optical means. This constitutes one of the bases of optical communications, allowing the installation of long-distance systems. Short light pulses are used as bits of information in such systems. Nowadays, sub-nanosecond light pulses commonly propagate in optical fibers, as top-level commercial systems are running at 10-40 Gbit/s. In competition with wavelength multiplexing systems that already reach data rates of several Tbit/s [1], it is expected that time-multiplexing systems will appear in a close future, carrying short pulses corresponding to transmission rates higher than $100 \mathrm{Gbit} / \mathrm{s}$. This requires detectors of light whose band width exceeds $100 \mathrm{GHz}$, i.e. whose time response is of the order of a few picoseconds. Beside the telecommunication domain, which certainly constitutes the main market for such a technology, ultrafast photodetectors 
are employed in many areas of technology and physics, like high speed electronics, triggering of electronics circuits, optically-fed phase-array radar antennae, generation of short electrical events, antennae for terahertz spectroscopy.

Today, the most efficient light-electricity converters are semiconductor (SC) devices. The absorption of incoming photons excites electrons from the valence band or from traps to the conduction band. This phenomenon first creates a population of dipoles in the SC, changing the polarization of the material and thus leading to a displacement current. Then, under a bias voltage, the photocarriers are accelerated and produce the conduction current. The current exists until all the photogenerated carriers get captured, recombine or reach the device electrodes. As the energy is given not only by the light pulse but also by the bias voltage, the efficiency of photoconducting devices surpasses those of other devices based for example on nonlinear optical phenomena.

The time response of ultrafast SC detectors is limited by either the carrier lifetime, the carrier transit time in the device, or the time constant of the whole electronic circuit that includes the detector. The photoexcitation of the carriers can be supposed as being instantaneous. The uncertainty principle $\Delta E \Delta t<h$ leads to a band-to-band excitation duration $\Delta t<2$ fs for $\Delta E \approx 2 \mathrm{eV}$, i.e. for an $\mathrm{SC}$ with the band gap in the visible [2]. The main limitation due to the material is thus the carrier recombination time. The recombination process can be strongly fastened by adding defects in the material, which act as efficient traps for free carriers. Today, the most popular materials are low-temperature grown (LTG) $\mathrm{SCs}$ that are non-stoichiometric. The species in excess play the role of traps. The carrier lifetime can be extremely short: values smaller than 50 fs have been reported in LTG-GaAs [3]. Alternative materials are polycrystalline, ion-implanted, damaged and amorphous SCs layers. In order to obtain high photoinduced current density, the carriers must exhibit a high mobility, favoring III-V or II-VI compounds as compared to column IV SC ( $\mathrm{Si}, \mathrm{Ge}$ ). Typically, electron mobility in LTG-GaAs is within the range $1000-2000 \mathrm{~cm}^{2} /(\mathrm{V} \mathrm{s})$ [4], smaller than the value for semi-insulating GaAs (SI-GaAs), but much higher than in $\mathrm{Si}$, CdTe, and $\mathrm{InP}$ [5].

Apart from the material properties, the geometry of photodetection devices has to be optimized in order to achieve high conversion efficiency and ultrashort device time response. Today, the fastest device is a photoswitch made of two metallic parallel strips, serving as electrodes, deposited over a fast SC, known as the Auston switch [6]. Its response time benefits from the small photoswitch capacity and electrical pulses as short as $200 \mathrm{fs}$ have been generated [7]. However, its efficiency is weak but could be increased using metal-SC-metal (MSM) diode geometry. MSM diode electrodes are interdigitated metallic fingers deposited over the SC. The illuminated area is wider than in the Auston switch but the MSM capacity is relatively large, increasing the time response of the diode. Other devices, like pin photodiodes [8,9] or traveling wave detectors [10], exhibit also remarkable performances. The Auston switches, as well as MSM, Schottky and pin diodes, 
generate not only current or voltage pulses delivered to the connected circuit, but also radiate electromagnetic transients in the free space, because the device can be pictured as a dipole. When the switch is photoexcited, its dipolar moment varies abruptly giving rise to a radiation whose far field is proportional to the time derivative of the current density flowing through the device [11, 12]. Fast devices, like LTG-GaAs photoswitches, produce sub-ps electromagnetic bursts, whose spectrum spreads typically from some tens of $\mathrm{GHz}$ up to several $\mathrm{THz}$. The best value reported is larger than $60 \mathrm{THz}$ [13], corresponding to wavelengths ranging from $5 \mu \mathrm{m}$ up to $1 \mathrm{~cm}$. These devices, which produce the widest controllable electromagnetic spectrum, are named $\mathrm{THz}$ antennae. They have opened new fields of investigation of the far infrared, with remarkable results in spectroscopy, sensing, and imaging [14].

In this review paper, we will focus our attention on ultrafast photoswitches and related ultrafast SC. We will first recall what are the SC physical parameters required to manufacture ultrafast switches. Then we will describe the basic physical properties of such SC, in terms of optical and electrical properties. We will present several selected materials among the most popular, such as LTG SCs. Finally, we will briefly describe a few applications of these ultrafast materials and photoswitches, like antennae for $\mathrm{THz}$ time-domain spectroscopy and nonlinear optical devices. Ultrafast processes in low-dimension devices and materials like quantum dots or quantum wells are not treated in this paper. The interested reader is referred to the complete review paper by Nolte [15].

\section{Material requirements}

A photoswitch is generally made of a highly resistive SC wafer on top of which metallic electrodes are evaporated. The gap between the electrodes becomes conductive via the photogeneration of carriers. The photoswitch is externally biased under a voltage $U$. The whole electrical circuit includes the photoswitch, the voltage supply, and a resistance $R_{\mathrm{c}}$ that could be either an external load, the contact resistance of the electrodes, or the impedance of the microwave line formed by the electrodes, if any. As the expected electrical signals are very short, the electrical behavior of the whole circuit must be treated within an electromagnetic theory. However the task is difficult as one should calculate both the carrier dynamics in the SC and the resulting current signal in the whole circuit. It is easier first to consider the photoswitch as a component for which one can compute the optoelectronical response, and then to introduce its equivalent electrical model in the circuit.

Without illumination, charges are stocked at the electrodes, which behave as a capacitor. Due to the finite conductivity of the SC material, one observes a weak dark current. Thus the equivalent model for the non-excited switch is a dark resistance $R_{\text {dark }}$ in parallel with a capacity $C$. Under illumination, the SC 
shows a strong photoconductivity, which adds to the dark conductivity. Therefore, in terms of resistance, the photoswitch is equivalent to a photovarying resistance

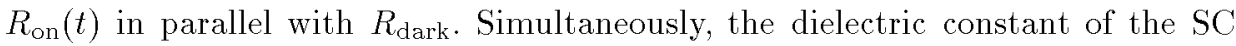
varies, because of the photocarrier population excited in the conduction band, and consequently so does the switch capacity. Neglecting in a first approach the propagation effect, the electrical signal in the circuit obeys the relation

$$
C(t) \frac{\mathrm{d} V(t)}{\mathrm{d} t}+V(t) \frac{\mathrm{d} C(t)}{\mathrm{d} t}+V(t)\left(\frac{1}{R_{\mathrm{c}}}+\frac{1}{R_{\mathrm{dark}}}+\frac{1}{R_{\mathrm{on}}(t)}\right)=\frac{U}{R_{\mathrm{c}}},
$$

where $V(t)$ is the voltage at the switch. Both $C(t)$ and $R_{\text {on }}(t)$ depend on the densities $n(t)$ of free electrons and $p(t)$ of free holes. For example, $R_{\mathrm{on}} \propto \frac{V(t)}{e\left[n(t) v_{\mathrm{e}}+p(t) v_{\mathrm{h}}\right]}$, where $v_{\mathrm{e}}$ and $v_{\mathrm{h}}$ are respectively the electron and hole velocities. The photogenerated carrier population dynamics is usually described with a population rate model [16]:

$$
\begin{aligned}
& \frac{\mathrm{d} n(t, r)}{\mathrm{d} t}=g(t, r)-\frac{n(t, r)}{\tau_{\mathrm{e}}}+\boldsymbol{\nabla} \cdot\left[D_{n} \boldsymbol{\nabla} \cdot n(t, \boldsymbol{r})+\mu_{\mathrm{e}} n(t, r) \boldsymbol{E}(t, r)\right], \\
& \frac{\mathrm{d} p(t, \boldsymbol{r})}{\mathrm{d} t}=g(t, r)-\frac{p(t, \boldsymbol{r})}{\tau_{\mathrm{h}}}+\boldsymbol{\nabla} \cdot\left[D_{p} \boldsymbol{\nabla} \cdot p(t, \boldsymbol{r})-\mu_{\mathrm{h}} p(t, \boldsymbol{r}) \boldsymbol{E}(t, \boldsymbol{r})\right],
\end{aligned}
$$

where $g(t, r)$ is the photogeneration rate, $\tau_{\mathrm{e}}, \mu_{\mathrm{e}}, D_{\mathrm{e}}$ and $\tau_{\mathrm{h}}, \mu_{\mathrm{h}}, D_{\mathrm{h}}$ are respectively the electron and hole lifetimes, mobilities and diffusion coefficients. The two carrier populations interact through the Poisson law, giving rise to a screening of the applied field

$$
\boldsymbol{\nabla} \cdot \boldsymbol{E}(t, r)=\frac{e}{\varepsilon}[p(t, r)-n(t, r)] .
$$

This set of coupled nonlinear differential equations is solved numerically with finite differential time domain (FDTD) methods $[16,17]$. In general, the generated pulse shows a fast rise time, which is mostly limited by the incoming laser pulse duration. The decay exhibits usually two regimes, a fast one due to the electrons, which is limited either by the electron lifetime, the electron transit time, or the $R C$ time constant of the whole circuit [18]. The second one appears as a longer tail due to the holes. When using ultrafast materials, the most limiting parameter is the $R C$ time constant of the circuit. The peak amplitude of the pulse is proportional to the carrier mobility, the bias voltage, and the laser power. The signal contrast, i.e. peak amplitude over background level, is maximum when the dark current is minimized, that is when highly resistive materials are used.

It ends up that the ideal SC material for ultrafast photoswitching purposes should exhibit high free carrier mobility and ultrashort lifetime, high resistivity, and high electrical field breakdown.

Let us notice that, when dealing with low intensity signals or with ultrafast switches (pulse duration comparable to the carrier scattering time), the above model is no more suitable as averaging currents over long times and large number 
of carriers is impossible. One should employ statistical techniques, like the Monte Carlo one $[18,19]$, which nevertheless lead to overall results similar to those given by the simple model.

\section{Material properties}

\subsection{Lifetime}

The carrier lifetime is reduced when carriers are trapped by centers in the $\mathrm{SC}$, like defects, impurities, and dislocations. For low injection regime, the lifetime is given by the Shockley-Read-Hall theory: $\tau_{c}=\frac{1}{\sigma_{c} N_{\text {trap }} v_{\text {th }}}$, where $v_{\text {th }}$ is the thermal velocity of the carriers, $\sigma_{\mathrm{c}}$ is the capture cross-section and $N_{\text {trap }}$ - the trap center density. Trapping centers can be created in SC materials by ionic implantation, radiation damaging, growth of non-stoichiometric layers at a low temperature, or growth of polycrystalline and amorphous layers.

\subsection{Mobility}

The carrier mobility is proportional to the free carrier scattering time. The scattering process results from collisions either with impurities or with phonons. At room temperature, in high mobility crystals like GaAs, the phonons are the main cause of scattering. As the mobility varies inversely as the square root of the carriers effective mass [20], it is preferable to employ low effective mass materials, such as III-V or II-VI compounds. In engineered ultrafast materials like LTG-GaAs, the density of defects and impurities is high enough to contribute to the scattering process. The mobility, in materials for which elastic scattering from neutral defects occurs, was estimated by Ersingoy to vary inversely to the defect density [21]. The mobility in such materials could be reduced by a factor larger than 10 as compared to the perfect bulk crystal.

In ultrafast SC, the evaluation of the mobility is a difficult task leading to different values reported for the same material (see Table I). Many reasons explain the dispersion of the results. In SC with sub-ps carrier lifetime, the conduction of the carriers takes place during a time comparable with the carrier scattering time. This means that before the excitation of a phonon, the carrier displacement is ballistic, and then it tends towards an ohmic behavior [22]. In GaAs, excitation of phonons occurs within 100-200 fs [23, 22]. On the other hand, the non-thermal behavior of the free carrier at very short time after excitation slows down the mobility [24]. In addition, when the SC excitation is performed with high-energy photons, relaxation to the $L$ valley (small mobility) and then to the $\Gamma$ valley (high mobility) can also be observed.

Therefore, a great care should be taken when measuring the carrier mobility. The classical van der Pauw method gives the "static" Hall mobility, but not the transient mobility. Methods based on the integrated efficiency of photoswitches [25] 
TABLE $\mathbf{I}$

Electron mobility and lifetime in selected ultrafast SCs.

\begin{tabular}{|c|c|c|c|c|c|}
\hline Material & $\begin{array}{c}\text { Material } \\
\text { parameters }\end{array}$ & $\begin{array}{c}\text { Mobility } \\
{\left[\mathrm{cm}^{2} /(\mathrm{V} \mathrm{s})\right]}\end{array}$ & $\begin{array}{c}\tau_{\text {scatter }} \\
{[\mathrm{fs}]}\end{array}$ & $\begin{array}{c}\text { Tifetime } \\
{[\mathrm{ps}]}\end{array}$ & Remarks \\
\hline RD-SOS & oxygen & 422 & 48 & 4 & $\begin{array}{l}\text { optical pump } \\
\text { - THz probe } \\
\text { experiments }[26]\end{array}$ \\
\hline RD-SOS & $\begin{array}{c}\text { argon } \\
\text { oxygen }\end{array}$ & $\begin{array}{c}10-100[27] \\
29[28] \\
360[29]\end{array}$ & & $\begin{array}{l}500[28] \\
600[30]\end{array}$ & $\begin{array}{c}\text { integrated } \\
\text { photoswitch } \\
\text { efficiency }\end{array}$ \\
\hline SOS & & $200-400[31]$ & & & \\
\hline LTG-GaAs & & $2250-3000$ & & $280-660$ & $\begin{array}{c}\text { THz pulse } \\
\text { analysis [4] }\end{array}$ \\
\hline LTG-GaAs & $T_{\mathrm{g}}=200^{\circ} \mathrm{C}$ & 150 & & 300 & $\begin{array}{c}\text { integrated } \\
\text { photoswitch } \\
\text { efficiency [25] }\end{array}$ \\
\hline LT-GaAs & & 1650 & & 300 & $\begin{array}{c}\text { saturation } \\
\text { of } \mathrm{THz} \\
\text { emission [32] }\end{array}$ \\
\hline $\begin{array}{l}\text { ion-implanted } \\
\text { GaAs }\end{array}$ & & $0.5-2000[33]$ & & $200-5000$ & $\begin{array}{c}\text { Hall } \\
\text { mobility }[33]\end{array}$ \\
\hline $\begin{array}{l}\text { CdTe } \\
\text { polycrystalline }\end{array}$ & & $180[34]$ & & $450[34]$ & \\
\hline
\end{tabular}

may lead to underestimated mobility. It seems that methods based on the analysis of $\mathrm{THz}$ pulses [4] radiated by dipole antennae are more truthful. Table I gives the mobility, scattering and lifetimes in several selected materials, together with the method employed to measure the mobility.

\subsection{Electrical field breakdown}

The electrical breakdown occurs when carriers, accelerated by the bias field, acquire a large amount of kinetic energy and become able to ionize atoms through collisions. This a valanche process leads to an exponential increase in the free carrier density along the device. The device resistance does not obey the Ohm law, but the $I(V)$ curve shows a nonlinear behavior at high applied fields. In LTG materials, the conductivity at low electrical fields is due to charge hopping, while at high fields, thermoionic and field emissions from defect participate in the conductivity. Most of free carriers are captured by the high density of defects and thus cannot participate in the impact ionization, resulting in high electrical breakdown values. Luo et al. [35] have measured values varying from 80 to $320 \mathrm{kV} / \mathrm{cm}$ (to be compared 
with $10 \mathrm{kV} / \mathrm{cm}$ in SI-GaAs) when the growth temperature decreases from 300 to $200^{\circ} \mathrm{C}$. In beryllium doped LTG-GaAs, the breakdown field is even higher than in undoped LTG-GaAs $\left(530 \mathrm{kV} / \mathrm{cm}\right.$ for $[\mathrm{Be}]=5 \times 10^{18} \mathrm{~cm}^{-3}$ grown at $\left.280^{\circ} \mathrm{C}[36]\right)$. Annealing the samples at $600^{\circ} \mathrm{C}$ reduces the defect density and consequently the breakdown field rises up to $350 \mathrm{kV} / \mathrm{cm}[36]$.

\subsection{Dark resistivity}

The dark resistivity is a limiting factor in two cases. The first one is related to the band conductivity, which appears in small energy band gap materials. Indeed, the band gap width is too small to forbid thermal excitation of free carriers. This is the case in the infrared, for example with $1.55 \mu \mathrm{m}$ materials like InAs. In SCs with a large amount of defects, many of them are ionized and one observes a hopping-type dark conductivity that is characterized by a $T^{-1 / 4}$ dependence on temperature. As the charges hop from one defect to another one via tunneling assistance, dark conductivity varies as the defect density. Typically dark resistivity in LTG-GaAs is of the order of $5 \times 10^{3}-10^{4} \Omega \mathrm{cm}[35]$. Annealing reduces the number of arsenic antisites, which leads to a decrease in hopping conductivity.

\section{Selected ultrafast materials}

\subsection{Low-temperature grown materials}

Low-temperature grown III-V SCs are nowadays the most popular materials for ultrafast photoswitching. Due to the growth at a low substrate temperature $\left(200-300^{\circ} \mathrm{C}\right)$, the material exhibits a species in excess, which appears either as interstitial or antisite defect in the crystal structure. Moreover, vacancies of the other species are also observed [37]. In LTG-GaAs, the low-temperature growth leads to $\approx 1 \%$ excess arsenic when the layer is grown under arsine overpressure. The defect density $\left(\left[\mathrm{As}_{\mathrm{Ga}}\right]=10^{15}-10^{20} \mathrm{~cm}^{-3}\right.$ ) varies inversely with the growth temperature in the range $250-300^{\circ} \mathrm{C}[38]$. The carrier lifetime is a linear function of the defect density in the growth temperature range $200-300^{\circ} \mathrm{C}[25,4,39]$.

The crystallographic quality of the layers is greatly improved by a post-annealing at high temperature $\left(600-800^{\circ} \mathrm{C}\right)$. A large part of the arsenic in excess precipitates to form metallic clusters of $50-100 \mathrm{~nm}$ diameter. It is now admitted that the main traps for electrons are the ionized As antisites, $\mathrm{As}_{\mathrm{Ga}}^{+}$, which constitute $5-10 \%$ of the total antisites [38]. Holes are mostly captured by the gallium vacancies $V_{\mathrm{Ga}}$. The precipitates seem to play a minor role in the carrier lifetime since arsenic-implanted GaAs [40] or polycrystalline LTG-GaAs [41], without precipitates, behaves as LTG-GaAs. However this question is still under discussion [42]. The important point is thus to control the ionized As $\mathrm{Ga}_{\mathrm{Ga}}^{+}$density, i.e. the concentration of acceptors $\left(\mathrm{V}_{\mathrm{Ga}}\right)$ that compensate for As $\mathrm{s}_{\mathrm{Ga}}$. It depends strongly on the growth and annealing parameters, like the temperature and the beam equivalent pressure of the As and Ga fluxes. 
Doping LTG-GaAs with an acceptor like beryllium compensates the arsenic in excess, and thus gives an additional degree of freedom to control the $\mathrm{As}_{\mathrm{Ga}}^{+}$density. The second advantage of doping with small atoms like Be is the relaxation of the lattice stress induced by the arsenic in excess. Thicker good quality layers may be grown [43]. However, Be doping must remain at a low level to prevent electrical conduction in the material. It is expected that $\mathrm{As}_{\mathrm{Ga}}^{+}$density will increase and consequently the carrier lifetime will decrease proportionally to the Be concentration. However, this is not experimentally observed, neither for the $\mathrm{As}_{\mathrm{Ga}}^{+}$density [44] nor for the carrier lifetime that first increases with Be concentration and then abruptly decreases over $[\mathrm{Be}]=10^{19} \mathrm{~cm}^{-3}[45]$. It is possible that some of Be atoms occupy the place of $\mathrm{V}_{\mathrm{Ga}}$ in the lattice of LTG-GaAs [43]. As each $\mathrm{V}_{\mathrm{Ga}}$ can keep 3 electrons while Be keeps only one, introducing Be reduces $\mathrm{As}_{\mathrm{Ga}}^{+}$density and increases the carrier lifetime. This phenomenon occurs until all the $\mathrm{V}_{\mathrm{Ga}}$ are filled with Be. For higher Be concentration, each additional Be atom compensates for one arsenic in excess, and thus $\mathrm{As}_{\mathrm{Ga}}^{+}$density increases, leading to very short carrier lifetimes at high Be concentrations.

Although the band gap energy of LTG-GaAs is $1.43 \mathrm{eV}\left(\lambda_{\text {gap }} \approx 0.87 \mu \mathrm{m}\right)$, attempts have been done to excite this material at the telecommunication wavelength $(1.55 \mu \mathrm{m}-0.8 \mathrm{eV})$ via two-photon absorption [46, 47]. The switching process is as fast as in a single photon process, but the efficiency is of course much weaker. Mid-gap defect states may play a role in the excitation process [47]. A possible alternative LTG material for switching at $1.5 \mu \mathrm{m}$ is LTG-InGaAs [25, 48]. InAlAs [25] and CdTe [49] have also been grown at low temperature for photoswitching applications.

\subsection{Ion implanted materials}

Many materials have been implanted with different ions. The implantation with light ions damages the crystal lattice (proton in GaAs [50] and InP [51], or oxygen in silicon on sapphire (RD-SOS) $[30,26]$ ). Post-implantation thermal annealing reduces the lattice defect density [52], leading to higher carrier mobility but to longer carrier lifetime. In GaAs, implanted heavy ions behave as point defects, in a way similar to arsenic in excess in LTG-GaAs [40]. Many ions have been implanted in GaAs, such as As [53-55], Ga [56, 57], Si and O [58]. For applications in telecommunications, InGaAs has been implanted with $\mathrm{O}$ and $\mathrm{Ni}$ [59].

\subsection{Other materials}

In polycrystalline or amorphous materials, grain boundaries may form defects. Thus the carrier lifetime could be very short but the charge transport occurs in the extended band tails (Urbach tail) hence resulting in a low mobility. Nonetheless ultrafast devices have been fabricated with such materials. Amorphous silicon [60] and polycrystalline silicon [61] have been among the first studied ultrafast $\mathrm{SC}$. Amorphous GaAs is usually grown over dielectric substrate like $\mathrm{MgO}$ [62] and 
it shows sub-picosecond carrier lifetime [62,63]. Polycrystalline LTG-GaAs exhibits similar performance [41].

\section{Applications}

\subsection{Photoswitches}

Photoswitches are made of two electrodes evaporated over a highly resistive ultrafast SC. Usually, the electrodes constitute either a strip or a coplanar microwave waveguide capable of supporting broad band propagation. The ultimate devices are designed on membrane substrates whose band width exceeds the $\mathrm{THz}$ threshold [64]. The signals are measured by electrooptic or photoconductive sampling techniques [65]. The fastest record (200 fs FWHM) has been published by Keil and Dykaar [7], who measured the generated electrical field at the location of optical excitation. More commonly, the electrical signal is measured along the microwave waveguide that serves as electrode. Typically LTG-GaAs switches exhibit picosecond response time $[25,66,67]$. Devices with wide gap between electrodes switch kilovolts in a few picoseconds [68]. The efficiency of the dipolar switches is rather weak (peak amplitude $\approx 1 \mathrm{~mA} / \mathrm{W}[69,55]$ ). To increase the conversion efficiency, it is preferable to employ ultrafast MSM diodes, whose efficiency is of the order of $0.1 \mathrm{~A} / \mathrm{W}[70]$. Their response time is somewhat longer, limited by the $R C$ time constant of the device. Nevertheless, Kordos et al. [71] have produced $650 \mathrm{fs}$ duration pulses with a $2 \mu \mathrm{m}$ interfinger LTG-GaAs MSM diode. Compact picosecond photoswitches are nicely used to fabricate photoconducting probes [72-75] that detect and measure high frequency and/or ultrafast electrical signals in high performance circuits, without introducing much perturbation.

\subsection{THz antennae}

$\mathrm{THz}$ antennae are dipole-like switches. The gap between the two electrodes is generally much smaller $(\approx 10 \mu \mathrm{m})$ than the radiated sub-millimeter wavelengths, justifying the dipolar approximation, even if quadrupolar terms in the radiated field have been reported [76]. Nevertheless, the dipolar behavior of the radiated field is also observed with wide gap antennae used in powerful systems [77]. In the dipolar approximation, the far field radiated by a time-varying dipole $P(t)$ is given by the time derivative of the current $J(t)$ flowing in the switch

$$
E_{\mathrm{THz}} \propto \frac{\mathrm{d}^{2} P}{\mathrm{~d} t^{2}}=\frac{\mathrm{d} J}{\mathrm{~d} t}=\frac{\mathrm{d}}{\mathrm{d} t}\left[e\left(n_{\mathrm{h}} v_{\mathrm{h}}-n_{\mathrm{e}} v_{\mathrm{e}}\right)\right]
$$

Therefore, the THz field amplitude will be very large if both the density and mobility of photocarriers are high and the current pulse is short. The THz dipole is usually attached to an antenna, in order both to enhance the coupling of the wave to free space and to make the $\mathrm{THz}$ beam almost parallel. The most popular arrangement [78] is a photoswitch manufactured over a highly resistive substrate 
and attached to a hyper-hemispherical dome, both being located at the focus of a parabolic mirror. The dipole structure shows the widest band width, but its gain is rather low. It can be slightly improved by designing sharp edge electrodes [79]. If one desires to optimize the efficiency for a limited frequency range, it is preferable to use dedicated high-gain antennae, like spiral antennae [80]. It must be noticed that nonuniform illumination of the photoswitch gap produces $\mathrm{THz}$ pulses with enhanced amplitude [81].

The $\mathrm{THz}$ field can be detected by photoconduction in an ultrafast switch. Let an optical pulse generates carriers in an unbiased switch: there is no current flowing in the switch because of the absence of field. But, if simultaneously the field of the $\mathrm{THz}$ radiation illuminates the switch, the photocarriers are accelerated by this field and a current is created. Contrary to optical detectors that are sensible to the intensity of the light beam, this $\mathrm{THz}$ detector shows the unique characteristic of delivering signals proportional to the $\mathrm{THz}$ field amplitude. The temporal shape of the signal is measured by sampling techniques: the same laser beam is used to excite both emitter and receiver with an adjustable time delay between both. The detected signal writes

$$
i(\tau) \approx \int_{-\infty}^{+\infty} \int_{-\infty}^{+\infty} E_{\mathrm{THz}}\left(t^{\prime}-\tau\right) I_{\text {laser }}(t) \eta_{\text {receiver }}\left(t-t^{\prime}\right) \mathrm{d} t \mathrm{~d} t^{\prime},
$$

where $I_{\text {laser }}$ is the laser intensity that excites the receiver, and $\eta_{\text {receiver }}$ is the impulse time response of photocarriers in the receiver. A careful study $[82,83]$ of the preceding expression shows that the signal spectrum depends mostly on the carrier lifetime in the receiver and on the laser pulse duration, as observed experimentally [84]. Following this rule, $20 \mathrm{THz}$ waves were generated and detected, using 15 fs laser pulses, with LTG-GaAs photoconducting antennae [85], and subsequently $60 \mathrm{THz}$ using an improved shaker technique [13].

Nowadays, alternative time-domain optoelectronics systems compete the photoconductive antennae. $\mathrm{THz}$ pulses can be generated by excitation of carriers at an SC surface [86]: the surface edge exhibits a decrease in the electronic density, which leads to a bending of the energy bands and thus to an intrinsic surface field. Carriers photogenerated at the SC surface are accelerated in the surface region and radiate a $\mathrm{THz}$ signal directed mostly along the surface, as the equivalent dipole is normal to the surface. When large waist beams are employed, the THz beam direction is given by quasi-Fresnel laws [86]. The $\mathrm{THz}$ pulse duration (sub-ps) is given by the electron transit time through the surface region and the generated signals are comparable to those achieved with photoswitches. The most efficient material for surface generation is InAlAs [87]. However, the badly controlled shape of the THz beam makes difficult the analysis of some experimental data like in $\mathrm{THz}$ time-domain spectroscopy.

$\mathrm{THz}$ pulses may also be generated by optical rectification in a nonlinear crystal [88]. Optical rectification corresponds to frequency difference in a nonlinear crystal showing no center of symmetry. The origin of this phenomenon is linked 
to the nonparabolic molecular potential wells experienced by the electrons. In theory, as non-resonant nonlinear effects are almost instantaneous, this technique is capable of producing the widest $\mathrm{THz}$ spectra and, as a matter of fact, ultrawide spectra spreading have been reported [89-91]. However, two factors limit the performance of $\mathrm{THz}$ sources based on optical rectification. First, excitation of optical phonons in the organic $[92,93]$ or ferroelectric [90] nonlinear crystals leads to weak efficiency zones in the generated spectra, where the signal drops to zero. Secondly, high efficiency occurs only when the laser and $\mathrm{THz}$ beams are phase-matched, in order to cumulate the effect of generation all along the crystal thickness. This requirement is difficult to meet because of dispersion. ZnTe [94] shows a weak difference of the optical and microwaves indices, allowing approximate phase-matching. Generally, this problem is overcome by using thin crystals. The detection of $\mathrm{THz}$ waves could be achieved in the same type of crystals by electrooptic sampling [95, 96]. The incoming $\mathrm{THz}$ field induces a birefringence in the crystal. The polarization of an optical probe beam, transmitted through the crystal, rotates because of the birefringence. The measure of the polarization rotation allows one to determine the $\mathrm{THz}$ field amplitude and direction. This technique shows the same advantage and drawback as $\mathrm{THz}$ generation by optical rectification, that is extremely large band width, but smaller efficiency than photoswitch devices. This last point makes photoswitch $\mathrm{THz}$ antennae preferable to build up sensitive $\mathrm{THz}$ time-domain spectroscopy setup, as, excited by the same laser power, electrooptic systems are noisier [97].

\subsection{Nonlinear devices}

Optical nonlinearities in semiconductors are already used in various devices. In the sub-band-gap wavelength range, bound-electron induced refractive index changes in semiconductors are somewhat larger than in dielectric crystals. However, multiple-quantum wells and other SC optical devices working in the material transparency region have rather narrow dynamic range because two-photon absorption limits the optical power in the semiconductor and the free carriers generated in this process recombine on a rather long timescale. On the other hand, band-to-band absorption induced nonlinearities, mainly due, at room temperature, to band filling effect, are larger by several orders of magnitude as compared to bound electron contribution (Table II). In this respect, ultrafast SCs, like LTG-GaAs and other non-stoichiometric SCs, offer at least two advantages over traditional semiconductors. The short lifetimes of the non-equilibrium carriers effectively remove the limitations due to multiphoton absorption and lead to ultrafast nonlinear interactions (see Table II).

As yet, there are known only several nonlinear optical devices based on LTG-GaAs and related non-stoichiometric materials, with SESAM [105] as the most successful of them. SESAM is a Bragg reflector mirror in which a layer is made of LTG-GaAs, and whose reflectivity exhibits a strong nonlinearity due to 
TABLE II

Optical Kerr effect or band-to-band induced index change in various materials [5].

\begin{tabular}{c|c|c|c|c}
\hline \hline Material & $\begin{array}{c}n_{2} \\
{\left[\mathrm{~cm}^{2} / \mathrm{kW}\right]}\end{array}$ & Physical origin & $\begin{array}{c}\Delta n \\
\left(\text { at } 6 \mathrm{GW} / \mathrm{cm}^{2}\right)\end{array}$ & Ref. \\
\hline LT-GaAs & $\approx 10^{-9}$ & band to band absorption & $6 \times 10^{-3}$ & {$[98]$} \\
$\mathrm{GaAs}$ & $\approx 10^{-12}$ & bound electrons & $6 \times 10^{-6}$ & {$[99]$} \\
$\mathrm{GaAs}$ & $\approx 10^{-9}$ & band to band absorption & $6 \times 10^{-3}$ & {$[100]$} \\
$\mathrm{InSb}$ at $10 \mu \mathrm{m}$ & $2 \times 10^{-8}$ & band to band absorption & 0.12 & {$[101]$} \\
$\mathrm{MNA}_{\text {(organics }}$ & $2.5 \times 10^{-10}$ & delocalized $\pi$ electrons & $1.5 \times 10^{-3}$ & {$[102]$} \\
$\mathrm{SiO}_{2}$ glass & $3 \times 10^{-13}$ & bound electrons & $1.8 \times 10^{-6}$ & {$[103]$} \\
$\mathrm{SiO}_{2}-\mathrm{PbO}$ glass & $\approx 5 \times 10^{-12}$ & bound electrons & $30 \times 10^{-6}$ & {$[104]$}
\end{tabular}

band filling in the LTG-GaAs layer. The component is located in a laser cavity, in which it serves both as the $100 \%$ mirror of the cavity and as saturable absorber component: SESAM mode-locked lasers can produce pulses of less than 10 ps duration [106]. Besides SESAM, LTG-InGaAs layer grown on top of a Bragg reflector as a part of antiresonant structure was used in the realization of an ultrafast all-optical switch [107]. Although in majority of such efforts the effect of optical nonlinearity in LTG material should be enhanced by placing this layer into an interferometric structure, even a free-standing LTG-GaAs layer was already successfully used for achieving of the mode-locking in a laser [108]. Another prospective field for nonlinear optical applications of the non-stoichiometric semiconductors is concerned with photorefractive devices exploiting ultrafast recharging of the deep centers in the band gap [15]: among others, four-wave-mixing and dynamic holography experiments have been reported [15].

One has to point out that the physical characteristics of LTG-GaAs and related materials relevant for the optical devices are by far less studied as compared with the materials used for the manufacture of photoconductive switches. Growth of LTG materials with band gap smaller than in GaAs is one of the key problems, but there are large uncertainties regarding nonsaturating optical losses and trap emptying times that have to be solved.

\section{Conclusion}

Ultrafast optoelectronics is a very interesting area of research. The optical generation of sub-picosecond electrical signals has opened the field to numerous applications, from fundamental solid state physics, to ultrafast electronics and biological studies. The last decade has lead to prodigious progress, and nowadays sub-picosecond electrical pulses are commonly generated by many groups. Photoconducting switches are still the most efficient devices, but they are competing by alternative techniques, like electrooptics, and thus it is necessary to improve 
the performance of such ultrafast switches. This can be achieved by growing engineered materials, in which all the carrier dynamics and optoelectrical parameters are well controlled. Works performed on LTG-GaAs and related compounds are a nice example of how the materials can be adapted to the device requirements and how they can be engineered.

The progress of ultrafast optoelectronics will certainly follow three complementary ways:

- The fabrication of compact systems using laser diodes, which will allow "mass-production" at realistic costs and thus development of applications for the consumers.

- The manufacture of very high performance systems that include basic components like ultrafast photoswitches. Terahertz or terabit/s optoelectronics circuits (sample and hold circuits [109], logical gates [110, 111], terabits/s demultiplexing modules [112]) have already been made and tested.

- The design of new ultrafast components, based on magnetooptic effects [113] or on spin-polarized electrical transport [114], opening the door to "spintronics".

It is expected that the 100 femtosecond threshold will be broken during the next decade, and that components will deliver electrical transients of a few tens of femtosecond duration. No doubt that ultrafast optoelectronics will continue to produce amazing results for several years!

\section{Acknowledgments}

I would like to thank Professors B. Kowalski and M. Kamińska, who gave me the opportunity to present this review on ultrafast photoswitches at the Jaszowiec 2002 summer school. Critical reading of the manuscript by my co-worker Dr. J.-F. Roux is acknowledged.

\section{References}

[1] See for example O. Graydon, Opto and Laser Europe 96, 31 (2002).

[2] W.M. Robertson, Optoelectronics Techniques for Microwaves and Millimeter Waves Engineering, Artech House, Norwood 1995.

[3] S. Marcinkevicius, A. Krotkus, R. Viselga, U. Olin, C. Jagadish, Semicond. Sci. Technol. 12, 396 (1997).

[4] H. Nemec, A. Pashkin, P. Kuzel, M. Khazan, S. Schnüll, I. Wilke, J. Appl. Phys. 90, 1303 (2001).

[5] J.-L. Coutaz, A. Krotkus, in: Proc. 3rd Symp. on Non-Stoichiometric III-V Compounds, Eds. S. Malzer, T. Marek, P. Kiesel, Physik Mikrostrukturierter Halbleiter, F. Alexander Universität Erlangen 23, 19 (2001).

[6] D. Auston, Appl. Phys. Lett. 26, 101 (1976); for a review see D.H. Auston, in: Semiconductors and Semimetals, Vol. 28, Ed. R.B. Marcus, Academic Press, San Diego 1993, p. 85. 
[7] U.D. Keil, D.R. Dykaar, IEEE J. Quantum Electron. 32, 1664 (1996).

[8] Y.G. Wey, K.S. Giboney, J.E. Bowers, M.J. Rodwell, P. Silvestre, P. Thiagarajan, G.Y. Robinson, IEEE Photonics Technol. Lett. 7, 412 (1993).

[9] A. Leitenstorfer, S. Hunsche, J. Shah, M.C. Nuss, W.H. Knox, Appl. Phys. Lett. 74, $1516(1999)$

[10] Y.-J. Chiu, S.B. Fleischer, D. Lasaosa, J.E. Bowers, Appl. Phys. Lett. 71, 2508 (1997).

[11] D.H. Auston, K.P. Chueng, P.R. Smith, Appl. Phys. Lett. 45, 284 (1984).

[12] Ch. Fattinger, D. Grischkowski, Appl. Phys. Lett. 53, 1480 (1988).

[13] S. Kono, M. Tani, K. Sakai, IEE Proc. - Optoelectronics 149, 105 (2002).

[14] See for example Terahertz Sources and Systems, Eds. R.E. Miles, P. Harrison, D. Lippens, NATO Science Series II, Vol. 27, Kluwer Academic Publishers, Dordrecht 2001.

[15] D.D. Nolte, J. Appl. Phys. 85, 6259 (1999).

[16] S.P. Wilson, A.B. Walker, Semicond. Sci. Technol. 12, 1265 (1997).

[17] X. Wang, R.J. Hwu, Microwave Opt. Technol. Lett. 1214, 204 (1997).

[18] S.Y. Chou, M.Y. Liu, IEEE J. Quantum Electron. 28, 2358 (1992).

[19] Y. Lu, Electron. Lett. 26, 445 (1990).

[20] J. Singh, Semiconductor Optoelectronics, Mc Graw Hill, New York 1985.

[21] C. Ersingoy, Phys. Rev. 79, 1013 (1950).

[22] B.B. Hu, E.A. de Souza, W.H. Knox, J.E. Cunningham, M.C. Nuss, A.V. Kuznetsov, S.L. Chuang, Phys. Rev. Lett. 74, 1689 (1995).

[23] J.A. Kash, J.C. Tsang, J.M. Hvam, Phys. Rev. Lett. 54, 2151 (1985).

[24] J.T. Darrow, X.-C. Zhang, D.H. Auston, J.D. Morse, IEEE J. Quantum Electron. 28, 1607 (1992).

[25] S. Gupta, J.F. Whitaker, G.A. Mourou, IEEE J. Quantum Electron. 28, 2464 (1992).

[26] K.P.H. Lui, F.A. Hegmann, Appl. Phys. Lett. 78, 3478 (2001).

[27] D. Auston, in: Ultrashort Laser Pulses: Generation and Applications, 2nd ed., Ed. W. Kaiser, Springer, Berlin 1993, p. 188.

[28] P.R. Smith, D.H. Auston, M.C. Nuss, IEEE J. Quantum Electron. 24, 255 (1988).

[29] M. Roser, S.R. Clayton, P.R. de la Houssaye, G.A. Garcia, IEEE Trans. Electron Devices 39, 2665 (1992).

[30] F.E. Doany, D. Grischkowsky, C.-C. Chi, Appl. Phys. Lett. 50, 460 (1987).

[31] D. McLeod, Jr., D.A. Shields, J.E.A. Maurits, D.H. Forbes, J. Vac. Sci. Technol. A 7, 1322 (1989).

[32] P. Benicewicz, J.P. Roberts, A.J. Taylor, J. Opt. Soc. Am. B 11, 2533 (1994).

[33] A. Krotkus, S. Marcinkevicius, J. Jasinski, M. Kaminska, H.H. Tan, C. Jagadish, Appl. Phys. Lett. 66, 3304 (1995).

[34] M.C. Nuss, D.W. Kisker, P.R. Smith, T.E. Harvey, Appl. Phys. Lett. 54, 57 (1989).

[35] J.K. Luo, H. Thomas, D.V. Morgan, D. Westwood, R.H. Williams, Semicond. Sci. Technol. 9, 2199 (1994). 
[36] M.J. Cich, R. Zhao, Y. Park, P. Specht, E.R. Weber, Mater. Res. Soc. Symp. Proc. 570, 129 (1999).

[37] J. Gebauer, F. Bömer, R. Krause-Rehberg, T.E.M. Staab, W. Bauer-Kugelmann, G. Kögel, W. Triftshäuser, P. Specht, R.C. Lutz, E.R. Weber, M. Luysberg, J. Appl. Phys. 87, 8368 (2000).

[38] X. Liu, A. Prasad, J. Nishio, E.R. Weber, Z. Lilienthal-Weber, W. Walukiewicz, Appl. Phys. Lett. 67, 279 (1995).

[39] G.-R. Lin, T.-A. Liu, C.-L. Pan, Jpn. J. Appl. Phys. 40, 6239 (2001).

[40] S. Marcinkevicius, C. Jagadish, H.H. Tan, M. Kaminska, K. Korona, R. Adomavicius, A. Krotkus, Appl. Phys. Lett. 76, 1306 (2000).

[41] J.-F. Roux, J.-L. Coutaz, A. Krotkus, Appl. Phys. Lett. 74, 2462 (1999).

[42] M.H. Zhang, L.W. Guo, H.W. Li, W. Li, Q. Huang, C.L. Bao, J.M. Zhou, B.L. Liu, Z.Y. Xu, Y.H. Zhang, L.W. Lu, Phys. Rev. B 11, 6311 (2001).

[43] A. Krotkus, K. Bertulis, M. Kaminska, K. Korona, A. Wolos, J. Siegert, S. Marcinkevicius, J.-F. Roux, J.-L. Coutaz, IEE Proc. - Optoelectronics 149, 111 (2002).

[44] P. Specht, R.C. Lutz, R. Zhao, E.R. Weber, W.K. Liu, K. Bacher, F.J. Towner, T.R. Stewart, M. Luysberg, J. Vac. Sci. Technol. B 17, 1200 (1999).

[45] A. Krotkus, K. Bertulis, L. Dapkus, U. Olin, S. Marcinkevicius, Appl. Phys. Lett. 75, 3336 (1999).

[46] H. Erlig, S. Wang, T. Azfar, A. Udupa, H.R. Fetterman, D.C. Streit, Electron. Lett. 35, 173 (1999).

[47] M. Tani, K.-S. Lee, X.-C. Xhang, Appl. Phys. Lett. 77, 1396 (2000).

[48] L.F. Lester, K.C. Hwang, P. Ho, J. Mazurowski, J.M. Ballingall, J. Sutuff, S. Gupta, J. Whitaker, S.L. Williamson, IEEE Photonics Technol. Lett. 5, 511 (1993)

[49] N. Breuil, A. Ghis, A. Reineix, B. Jecko, A. Barthelemy, in: Proc. SPIE "Millimeter and Submillimeter Waves IV”, Ed. M.N. Afsar, Vol. 3465, 1998, p. 348.

[50] M.B. Johnson, T.C. McGill, N.G. Paulter, Appl. Phys. Lett. 54, 2424 (1989).

[51] R.A. Höpfel, Ch. Teiss, K.F. Lamprecht, Phys. Rev. B 53, 12581 (1996).

[52] Y. Kobayashi, M. Nakamura, T. Suzuki, Appl. Phys. Lett. 40, 1040 (1982).

[53] A. Krotkus, S. Marcinkevicius, J. Jasinski, M. Kaminska, H.H. Tan, C. Jagadish, Appl. Phys. Lett. 66, 3304 (1995).

[54] F. Ganikhanov, G.R. Lin, W.C. Chen, C.S. Chang, C.L. Pan, Appl. Phys. Lett. 66, 3465 (1995).

[55] G.-R. Lin, W.-C. Chen, C.-S. Chang, S.-C. Chao, K.-H. Wu, T.M. Hsu, W.C. Lee, C.-L. Pan, IEEE J. Quantum Electron. 34, 1740 (1998).

[56] H.H. Tan, C. Jagadish, K.P. Korona, J. Jasinski, M. Kaminska, R. Viselga, S. Marcinkevicius, A. Krotkus, IEEE J. Select. Top. Quantum Electron. 2, 636 (1996).

[57] C. Jagadish, H.H. Tan, J. Jasinski, M. Kaminska, M. Palczewska, A. Krotkus, S. Marcinkevicius, Appl. Phys. Lett. 67, 1724 (1995). 
[58] C. Jagadish, H.H. Tan, A. Krotkus, S. Marcinkevicius, K.P. Korona, M. Kaminska, Appl. Phys. Lett. 68, 2225 (1996).

[59] E.L. Delpon, J.-L. Oudar, N. Bouché, R. Raj, A. Shen, N. Stelmakh, J.-M. Lourtioz, Appl. Phys. Lett. 72, 759 (1998).

[60] D.H. Auston, P. Lavallard, N. Sol, D. Kaplan, Appl. Phys. Lett. 36, 66 (1980).

[61] C. Shu, B.B. Hu, X.-C. Zhang, P. Mei, E.S. Yang, Appl. Phys. Lett. 57, 64 (1990).

[62] C.J. Osbahr, B.H. Larsen, T. Holst, Y. Shen, S.R. Keiding, Appl. Phys. Lett. 74, 1892 (1999).

[63] T. Kiwa, I. Kawashima, S. Nashima, M. Hangyo, M. Tonouchi, Jpn. J. Appl. Phys. 39, $6304(2000)$.

[64] H. Cheng, J.F. Whitaker, T.M. Weller, L.P.B. Katehi, IEEE Microwave Guided Wave Lett. 4, 89 (1994).

[65] Special Issue on Optical Probing of Ultrafast Devices, Optical and Quantum Electronics 28 (1996).

[66] Y. Chen, S. Williamson, T. Brock, F.W. Smith, A.R. Calawa, Appl. Phys. Lett. 59, 1984 (1991).

[67] H.-H. Wang, P. Grenier, J.F. Whitaker, H. Fujioka, J. Jasinski, Z. Lilienthal-Weber, IEEE J. Select. Topics Quantum Electron. 2, 630 (1996).

[68] C.W. Siders, J.L.W. Siders, A.J. Taylor, S.-G. Park, M.R. Melloch, A.M. Weiner, Opt. Lett. 24, 241 (1999).

[69] M.Y. Frankel, J.F. Whitaker, G.A. Mourou, F.W. Smith, A.R. Calawa, IEEE Trans. Electron Devices 37, 2493 (1990).

[70] Y. Chen, S.L. Williamson, T. Bork, F.W. Smith, IEDM Tech. Dig., 417 (1991).

[71] P. Kordos, A. Förster, M. Marso, F. Rüders, Electron. Lett. 34, 119 (1998).

[72] J. Kim, S. Williamson, J. Nees, S. Wakana, J. Whitaker, Appl. Phys. Lett. 62, 2268 (1993).

[73] M.D. Weiss, M.H. Crites, E.W. Bryerton, J.F. Whitaker, Z. Popović, IEEE MTT 47, 2599 (1999).

[74] S. Weiss, D.F. Ogletree, D. Botkin, M. Salmeron, D.S. Chemla, Appl. Phys. Lett. 63, 2567 (1993).

[75] J.-R. Hwang, H.-J. Cheng, J.F. Whitaker, J.V. Rudd, Appl. Phys. Lett. 68, 1464 (1996).

[76] J.V. Rudd, J.L. Johnson, D.M. Mittleman, Opt. Lett. 25, 1556 (2000).

[77] E. Burdiato, J. Margolies, S. Jeong, J. Son, J. Bokor, IEEE J. Quantum Electron. 32, 1839 (1996).

[78] M. van Exter, Ch. Fattinger, D. Grischkowsky, Appl. Phys. Lett. 55, 337 (1989).

[79] I. Brener, D. Dykaar, A. Frommer, L.N. Pfeiffer, J. Lopata, J. Wynn, K. West, M.C. Nuss, Opt. Lett. 21, 1924 (1996).

[80] S. Verghese, K.A. McIntsoh, E.R. Brown, IEEE Trans. MTT 45, 1301 (1997).

[81] S.H. Lu, J.L. Li, J.S. Yu, S.F. Horng, C.C. Chi, Appl. Phys. Lett. 77, 3896 (2000).

[82] Z.S. Piao, M. Tani, K. Sakai, Proc. SPIE 3617, 49 (1999). 
[83] L. Duvillaret, F. Garet, J.-F. Roux, J.-L. Coutaz, IEEE J. Select. Top. Quantum Electron. 7, 615 (2001).

[84] M. Tani, S. Matsuura, K. Sakai, S. Nakashima, Appl. Opt. 36, 7853 (1997).

[85] S. Kono, M. Tani, P. Gu, K. Sakai, Appl. Phys. Lett. 77, 4104 (2000).

[86] X.-C. Xhang, D.H. Auston, J. Appl. Phys. 71, 326 (1992).

[87] P. Haring Bolivar, in: Semiconductor Quantum Electronics, Ed. A. Miller, M. Ebrahimzadeh, D.H. Finlayson, SUSSP Publications, Edinburgh 1999, p. 151.

[88] A. Rice, Y. Jin, X.F. Ma, X.-C. Xhang, D. Bliss, J. Larkin, M. Alexander, Appl. Phys. Lett. 64, 1324 (1994).

[89] A. Bonvalet, M. Joffre, J.-L. Martin, A. Migus, Appl. Phys. Lett. 67, 2907 (1995).

[90] P.Y. Han, X.-C. Zhang, Appl. Phys. Lett. 73, 1349 (1998).

[91] A. Leitenstorfer, S. Hunsche, J. Shah, M.C. Nuss, W.H. Knox, Appl. Phys. Lett. 74, 1517 (1999).

[92] M. Walther, K. Jensby, S.R. Keiding, H. Takahashi, H. Ito, Opt. Lett. 25, 911 (2000).

[93] H. Hashimoto, H. Takahashi, T. Yamada, K. Kuroyanagi, T. Kobayashi, J. Phys., Condens. Matter 13, L529 (2001).

[94] Q. Wu, X.-C. Zhang, Appl. Phys. Lett. 71, 1285 (1997).

[95] P. Uhd Jepsen, C. Winnewisser, M. Schall, V. Schya, S.R. Keiding, H. Helm, Phys. Rev. E 53, 3052 (1996).

[96] S. Kono, M. Tani, K. Sakai, Appl. Phys. Lett. 79, 898 (2001).

[97] P. Planken, H.-K. Nienhuys, H.J. Bakker, T. Wenckebach, J. Opt. Soc. Am. B 18, $313(2001)$.

[98] H.S. Loka, S.D. Benjamin, P.W.E. Smith, Opt. Commun. 155, 206 (1998).

[99] P.N. Robson, NATO ASI Series E 214, 231 (1992).

[100] C.K.N. Patel, R.E. Slusher, P.A. Fleury, Phys. Rev. Lett. 17, 1011 (1966).

[101] T.Y. Chang, Opt. Eng. 20, 220 (1981).

[102] M.J. Goodwin, R. Glenn, C. Trundle, I. Bennion, J. Opt. Soc. Am. B 5, 419 (1988).

[103] A. Owyoung, R.W. Hellwarth, N. George, Phys. Rev. B 5, 628 (1972).

[104] S.R. Friberg, P.W. Smith, IEEE J. Quantum Electron. 23, 2089 (1987).

[105] U. Keller, K.J. Weingarten, F.X. Körtner, D. Kopf, B. Braun, I.D. Jung, R. Fluck, C. Hönninger, N. Matuschek, J. Aus der Au, IEEE J. Select. Top. Quantum Electron. 2, 435 (1996).

[106] D.H. Sutter, G. Steinmeyer, L. Gallmann, N. Matuschek, F. Morier-Genoud, U. Keller, V. Scheuer, G. Angelow, T. Tschudi, Opt. Lett. 24, 631 (1999).

[107] H.S. Loka, P.W.E. Smith, IEEE Photon. Techn. Lett. 10, 1733 (1998).

[108] M. Leitner, P. Glas, T. Sandrock, M. Wrage, G. Apostolopoulos, A. Riedel, H. Kostial, J. Herfort, K.-J. Friedland, L. Daeweritz, Opt. Lett. 24, 1567 (1999).

[109] R. Urata, R. Takahashi, V.A. Sabnis, D.A.B. Miller, J.S. Harris, IEEE Photonics Technol. Lett. 13, 717 (2001). 
[110] A. Hirano, H. Tsuda, H. Kobayashi, R. Takahashi, M. Asobe, K. Sato, K. Hagimoto, J. Lightwave Technol. 17, 873 (1999).

[111] M. Yoneyama, T. Shibata, E. Sano, Y. Kawamura, R. Takahashi, T. Enoki, T. Nagatsuma, M. Yaita, IEEE J. Quantum Electron. 33, 1308 (1997).

[112] H. Kobayashi, R. Takahashi, Y. Matsuoka, H. Iwamura, Electron. Lett. 34, 908 (1999).

[113] I. Tkach, K. Krambrock, C. Steen, P. Kiesel, J.M. Spaeth, Physica B 308, 749 (2001).

[114] M.J. Stevens, A.L. Smirl, R.D.R. Bhat, J.E. Sipe, H.M. van Driel, J. Appl. Phys. 91, $4382(2002)$. 